\title{
1-D SCHRÖDINGER OPERATORS WITH COULOMB-LIKE POTENTIALS
}

\author{
YURIY GOLOVATY
}

\begin{abstract}
We study the convergence of $1 \mathrm{D}$ Schrödinger operators $H_{\varepsilon}$ with the potentials which are regularizations of a class of pseudo-potentials having in particular the form

$$
\alpha \delta^{\prime}(x)+\beta \delta(x)+\gamma /|x| \text { or } \alpha \delta^{\prime}(x)+\beta \delta(x)+\gamma / x .
$$

The limit behaviour of $H_{\varepsilon}$ in the norm resolvent topology, as $\varepsilon \rightarrow 0$, essentially depends on a way of regularization of the Coulomb potential and the existence of zero-energy resonances for $\delta^{\prime}$-like potential. All possible limits are described in terms of point interactions at the origin. As a consequence of the convergence results, different kinds of $L^{\infty}(\mathbb{R})$-approximations to the even and odd Coulomb potentials, both penetrable and impenetrable in the limit, are constructed.
\end{abstract}

\section{INTRODUCTION AND MAIN RESUltS}

One-dimensional Schrödinger operators with the Coulomb potentials, the structure of their spectra and the question of penetrability of the Coulomb potentials have been the subject of several mathematical discussions [8-10], [11-14], starting with the work of Loudon [1]. These studies are related to the one-dimensional models of the hydrogen atom

$$
-\frac{d^{2} \psi}{d x^{2}}-\frac{\gamma}{|x|} \psi=E \psi, \quad-\frac{d^{2} \psi}{d x^{2}}+\frac{\gamma}{x} \psi=E \psi, \quad x \in \mathbb{R} .
$$

Since the potentials have singularities at the origin, the first derivative of wave function $\psi$ also has in general singularities as $x \rightarrow 0$, and therefore the wave function should be subject to some additional conditions at $x=0$. For these formal differential expressions, mathematics gives a large enough set of the boundary conditions associated with self-adjoint operators in $L^{2}(\mathbb{R})[11,15,16]$. The main issue here is a physically motivated choice of such conditions. We noticed that this problem has many common features with the problem of $\delta^{\prime}$-potential [17-24]. First of all, both the Coulomb potential and the $\delta^{\prime}$-potential are very sensitive to a way of their regularization. From a physical point of view, this means that there is no unique one-dimensional model of the hydrogen atom described by the pseudo-Hamiltonians in (1.1). However there are many different quantum systems with the Coulomb-like potentials that exhibit different physical properties.

2000 Mathematics Subject Classification. Primary 34L40, 34B09; Secondary 81Q10.

Key words and phrases. 1D Schrödinger operator, Coulomb potential, one-dimensional hydrogen atom, $\delta^{\prime}$-potential, scattering problem, penetrability of potential, point interaction. 
We study the norm resolvent convergence of Hamiltonians with the Coulomblike potentials perturbed by localized singular potentials. Assume that real-valued function $Q$ is locally integrable outside the origin and has an interior singularity at $x=0$, namely

$$
Q(x)=\left\{\begin{array}{llr}
\frac{q_{-}}{x}, & \text { if } & -a<x<0 \\
\frac{q_{+}}{x}, & \text { if } & 0<x<a
\end{array}\right.
$$

for some real constants $q_{-}, q_{+}$and $a>0$. We also suppose that $Q$ is bounded from below if $|x|>a$. Set

$$
Q_{\varepsilon}(x)=\left\{\begin{aligned}
Q(x), & \text { if }|x|>\varepsilon, \\
\frac{\ln \varepsilon}{\varepsilon} \varkappa\left(\frac{x}{\varepsilon}\right), & \text { if }|x|<\varepsilon,
\end{aligned}\right.
$$

where $\varkappa$ is a function belonging to $L^{\infty}(-1,1)$. Also let $U$ and $V$ be real-valued, measurable and bounded functions with compact supports. In additional, we suppose that their supports are contained in interval $\mathcal{I}=(-1,1)$. We study the convergence of Schrödinger operators

$$
H_{\varepsilon}=-\frac{d^{2}}{d x^{2}}+Q_{\varepsilon}(x)+\frac{1}{\varepsilon^{2}} U\left(\frac{x}{\varepsilon}\right)+\frac{1}{\varepsilon} V\left(\frac{x}{\varepsilon}\right)
$$

as the positive parameter $\varepsilon$ tends to zero. We hereafter interpret $\varepsilon^{-2} U\left(\varepsilon^{-1} \cdot\right)$ and $\varepsilon^{-1} V\left(\varepsilon^{-1} \cdot\right)$ as $\delta^{\prime}$-like and $\delta$-like potentials respectively, because

$$
\varepsilon^{-2} U\left(\varepsilon^{-1} x\right) \rightarrow \alpha \delta^{\prime}(x), \quad \varepsilon^{-1} V\left(\varepsilon^{-1} x\right) \rightarrow \beta \delta(x)
$$

in the sense of distributions as $\varepsilon \rightarrow 0$, provided $U$ is a function of zero-mean. In general, the potentials of $H_{\varepsilon}$ diverge, because we do not assume that $\int_{\mathbb{R}} U d x=0$.

Before stating our main result we introduce some notation. We say that the Schrödinger operator $-\frac{d^{2}}{d t^{2}}+U$ possesses a zero-energy resonance if there exists a non-trivial solution $h$ of the equation $-h^{\prime \prime}+U h=0$ that is bounded on the whole line. We call $h$ the half-bound state. We will also simply say that the potential $U$ is resonant and it possesses a half-bound state $h$. We set

$$
\theta=\frac{h(+\infty)}{h(-\infty)}
$$

where $h( \pm \infty)=\lim _{x \rightarrow \pm \infty} h(x)$. These limits exist, because the half-bound state is constant outside the support of $U$ as a bounded solution of equation $h^{\prime \prime}=0$. Moreover, both the values $h( \pm \infty)$ are different from zero. Since a half-bound state is defined up to a scalar factor, we fix half-bound state $h_{0}$ so that

$$
h_{0}(-\infty)=1, \quad h_{0}(+\infty)=\theta .
$$

Let us set

$$
\mu=\int_{\mathcal{I}} V h_{0}^{2} d x .
$$

We also introduce the spaces

$$
\mathcal{Q}_{ \pm}=\left\{\psi \in L^{2}\left(\mathbb{R}_{ \pm}\right): \psi, \psi^{\prime} \in A C_{\text {loc }}\left(\mathbb{R}_{ \pm}\right),-\psi^{\prime \prime}+Q \psi \in L^{2}\left(\mathbb{R}_{ \pm}\right)\right\}
$$

and denote by $\mathcal{Q}$ the space of $L^{2}(\mathbb{R})$-functions $\phi$ such that $\left.\phi\right|_{\mathbb{R}_{ \pm}} \in \mathcal{Q}_{ \pm}$. Here $A C_{\text {loc }}\left(\mathbb{R}_{ \pm}\right)$denotes the set of functions $\psi$ on $\mathbb{R}_{ \pm}$which are absolutely continuous 
on every compact subset of $\mathbb{R}_{ \pm}$. Note that the first derivative of $\phi \in \mathcal{Q}$ is in general undefined at the origin and has a logarithmic singularity at this point $[8,11,12]$.

We say self-adjoint operators $H_{\varepsilon}$ converge as $\varepsilon \rightarrow 0$ in the norm resolvent sense if the resolvents $\left(H_{\varepsilon}-\zeta\right)^{-1}$ converge in the uniform operator topology for all $\zeta \in \mathbb{C} \backslash \mathbb{R}$.

Our main result reads as follows.

Theorem 1. The operator family $H_{\varepsilon}$ given by (1.4) converges as $\varepsilon \rightarrow 0$ in the norm resolvent sense. If potential $U$ has a zero-energy resonance, the corresponding half-bound state $h_{0}$ satisfies (1.6) and

$$
\theta^{2} q_{+}-q_{-}=\int_{\mathcal{I}} x h_{0}^{2} d x
$$

then $H_{\varepsilon}$ converge to operator $\mathcal{H}$ that is defined by $\mathcal{H} \phi=-\phi^{\prime \prime}+Q \phi$ on functions $\phi$ in $\mathcal{Q}$, subject to the coupling conditions

$$
\begin{gathered}
\phi(+0)=\theta \phi(-0) \\
\lim _{x \rightarrow+0}\left(\theta \phi^{\prime}(x)-\phi^{\prime}(-x)-\left(\theta^{2} q_{+}-q_{-}\right) \phi(-0) \ln x\right)=\mu \phi(-0) .
\end{gathered}
$$

Otherwise, that is, if either (1.8) does not hold or else $U$ is not resonant, operators $H_{\varepsilon}$ converge to the direct sum $\mathcal{H}=\mathcal{D}_{-} \oplus \mathcal{D}_{+}$of the Dirichlet half-line Schrödinger operators $\mathcal{D}_{ \pm}=-\frac{d^{2}}{d x^{2}}+Q$ with domains $\operatorname{dom} \mathcal{D}_{ \pm}=\left\{\psi \in \mathcal{Q}_{ \pm}: \psi(0)=0\right\}$.

Moreover, in both the cases we have

$$
\left\|\left(H_{\varepsilon}-\zeta\right)^{-1}-(\mathcal{H}-\zeta)^{-1}\right\| \leq C \varepsilon^{1 / 4} .
$$

Remark 1. If a half-bound state $h$ is not normalized to unity at $x=-\infty$ as in (1.6), then (1.7) and (1.8) transform to read

$$
\mu=\frac{1}{|h(-\infty)|^{2}} \int_{\mathcal{I}} V h^{2} d x, \quad \theta^{2} q_{+}-q_{-}=\frac{1}{|h(-\infty)|^{2}} \int_{\mathcal{I}} x h^{2} d x
$$

Remark 2. Take note that point interactions (1.9) involve implicitly the regularizing function $\varkappa$ via condition (1.8), which describes a certain interaction of the $\delta^{\prime}$-like and the Coulomb-like potentials.

If we introduce notation $b_{ \pm}(\phi)=\lim _{x \rightarrow \pm 0}\left(\phi^{\prime}(x)-q_{ \pm} \phi( \pm 0) \ln |x|\right)$, then (1.9) can be written in the form

$$
\phi(+0)=\theta \phi(-0), \quad \theta b_{+}(\phi)-b_{-}(\phi)=\mu \phi(-0) .
$$

Taking into account the jump condition for $\phi$, we see that

$$
\begin{array}{r}
\theta b_{+}(\phi)-b_{-}(\phi)=\theta \lim _{x \rightarrow+0}\left(\phi^{\prime}(x)-q_{+} \phi(+0) \ln |x|\right)-\lim _{x \rightarrow-0}\left(\phi^{\prime}(x)-q_{-} \phi(-0) \ln |x|\right) \\
=\lim _{x \rightarrow+0}\left(\theta \phi^{\prime}(x)-\phi^{\prime}(-x)-\left(\theta q_{+} \phi(+0)-q_{-} \phi(-0)\right) \ln |x|\right) \\
=\lim _{x \rightarrow+0}\left(\theta \phi^{\prime}(x)-\phi^{\prime}(-x)-\left(\theta^{2} q_{+}-q_{-}\right) \phi(-0) \ln x\right) .
\end{array}
$$

Remark 3. In the case when $q_{-}=q_{+}=0$ and $\varkappa=0$, i.e., $Q$ has no singularity at the origin, the results of this article coincide with the results obtained in [1720], where the convergence of Hamiltonians with $\left(\alpha \delta^{\prime}+\beta \delta\right)$-like potentials was discussed. 
Now we give some consequences for scattering problems. Let us agree to say that the potentials in (1.4) are penetrable in the limit as $\varepsilon \rightarrow 0$ if the corresponding Schrödinger operators $H_{\varepsilon}$ converge to operator $\mathcal{H}$ associated with point interaction (1.9). If the operators converge to the direct sum $\mathcal{D}_{-} \oplus \mathcal{D}_{+}$, we say the potentials are opaque in the limit or asymptotically opaque.

Theorem 1 asserts that potentials $Q_{\varepsilon}+\varepsilon^{-2} U\left(\varepsilon^{-1} \cdot\right)+\varepsilon^{-1} V\left(\varepsilon^{-1} \cdot\right)$ are generally asymptotically opaque. However, for each potential $U$ that possesses a zero-energy resonance there exists a regularization of $Q$ having the form (1.3) such that condition (1.8) is fulfilled and hence the potentials are penetrable in the limit. It is also worth noting that resonant potentials are not something exotic, because for any $U$ of compact support there exists a discrete infinite set of real coupling constants $\alpha$ for which potential $\alpha U$ has a zero-energy resonance.

Coming back to the problem of penetrability of the Coulomb potentials, let us suppose that the potentials of $H_{\varepsilon}$ do not contain the $\delta^{\prime}$-like component, i.e., $H_{\varepsilon}=-\frac{d^{2}}{d x^{2}}+Q_{\varepsilon}+\varepsilon^{-1} V\left(\varepsilon^{-1} \cdot\right)$. We left the $\delta$-like potential in the Hamiltonian, because, as shown in the following theorem, $V$ has no direct influence on the penetrability in the limit.

Theorem 2. Potentials $Q_{\varepsilon}+\varepsilon^{-1} V\left(\varepsilon^{-1} \cdot\right)$ are penetrable in the limit as $\varepsilon \rightarrow 0$ if and only if $Q_{\varepsilon}$ converge in the sense of distributions. This is in turn true if and only if the condition

$$
q_{+}-q_{-}=\int_{\mathcal{I}} \varkappa d x
$$

holds. In the penetrable case, $H_{\varepsilon}$ converge to operator $\mathcal{H}$ associated with point interactions

$$
\phi(+0)=\phi(-0), \quad \lim _{x \rightarrow+0}\left(\phi^{\prime}(x)-\phi^{\prime}(-x)-\left(q_{+}-q_{-}\right) \phi(0) \ln x\right)=\beta \phi(0),
$$

where $\beta$ is the mean value of $V$.

\section{Coulomb-like potentials:}

PENETRABILITY AND OPAQUENESS IN THE LIMIT

In this section we will prove Theorem 2 and give some examples of the Coulomblike potentials $Q_{\varepsilon}$ that are penetrable and opaque in the limit.

2.1. Convergence of Coulomb-like potentials. Function $Q$ of the form (1.2) near the origin is nonintegrable and therefore mapping $C_{0}^{\infty}(\mathbb{R}) \ni \psi \mapsto \int_{\mathbb{R}} Q \psi d x$ is not a distribution. However we can find infinitely many functionals $q \in \mathcal{D}^{\prime}(\mathbb{R})$ which coincide with $Q$ outside the origin, i.e.,

$$
q(\psi)=\int_{\mathbb{R}} Q \psi d x \quad \text { for all } \psi \in C_{0}^{\infty}(\mathbb{R} \backslash\{0\}) .
$$

Among such functionals there exists the family $\mathcal{F}_{Q}$ of distributions with the lowest order of singularity. It is easy to check that each $q \in \mathcal{F}_{Q}$ is continuous in space $C_{0}^{0, \gamma}(\mathbb{R})$ of Hölder continuous functions of compact support, but $q$ is not continuous in $C_{0}^{0}(\mathbb{R})$. In this sense, $q$ is more singular than Dirac's $\delta$-function, but less singular than $\delta^{\prime}$-function. Moreover, if $q_{1}$ and $q_{2}$ belong to $\mathcal{F}_{Q}$, then $q_{2}-q_{1}=c \delta(x)$ for some complex constant $c$, and therefore $\mathcal{F}_{Q}=\left\{q_{1}+c \delta(x): c \in \mathbb{C}\right\}$. 

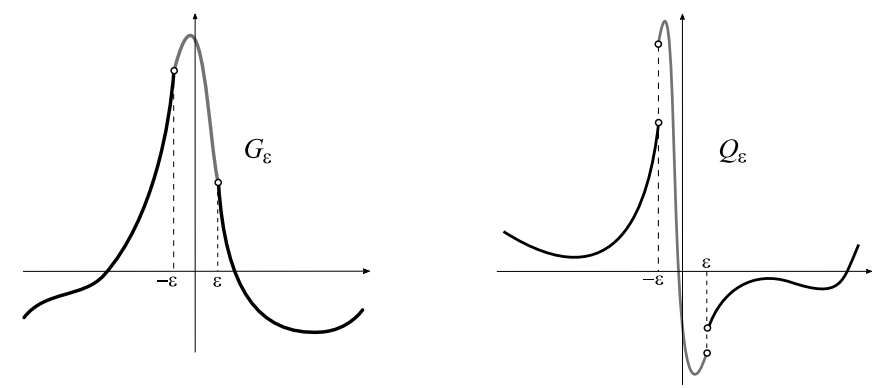

Figure 1. Plots of $G_{\varepsilon}$ and $Q_{\varepsilon}$

A word of explanation is necessary with regard to regularization of $Q$ given by (1.3). Here we use the analogy with formula $(\ln |x|)^{\prime}=\mathcal{P} \frac{1}{x}$. Suppose that $G$ is an antiderivative of $Q$ such that $G(x)=q_{-} \ln (-x)$ for $x \in(-a, 0)$ and $G(x)=q_{+} \ln x$ for $x \in(0, a)$. Function $G$ specifies a regular distribution on the line, because it belongs to $L_{l o c}^{1}(\mathbb{R})$. We set $g=G^{\prime}$, where $G^{\prime}$ is the derivative in the sense of distributions. Indeed, $g$ coincides with $Q$ outside the origin and $g \in \mathcal{F}_{Q}$. Let us now approximate $G$ in $\mathcal{D}^{\prime}(\mathbb{R})$ by the sequence of continuous functions

$$
G_{\varepsilon}(x)= \begin{cases}G(x), & \text { if }|x|>\varepsilon, \\ a\left(\frac{x}{\varepsilon}\right) \ln \varepsilon, & \text { if }|x|<\varepsilon,\end{cases}
$$

where $a$ is a $C^{1}$-function such that $a(-1)=q_{-}$and $a(1)=q_{+}$(see Fig. 1). Then distribution $g$ admits a regularization by $L_{l o c}^{1}(\mathbb{R})$-functions having the form

$$
Q_{\varepsilon}(x):=G_{\varepsilon}^{\prime}(x)= \begin{cases}Q(x), & \text { if }|x|>\varepsilon, \\ \frac{\ln \varepsilon}{\varepsilon} a^{\prime}\left(\frac{x}{\varepsilon}\right), & \text { if }|x|<\varepsilon .\end{cases}
$$

Lemma 1. A sequence $Q_{\varepsilon}$, given by (1.3), converges in $\mathcal{D}^{\prime}(\mathbb{R})$ if and only if condition (1.13) holds. Moreover, the limit distribution, if it exists, belongs to $\mathcal{F}_{Q}$.

Proof. For each $\psi \in C_{0}^{\infty}(\mathbb{R})$, we have

$$
\begin{aligned}
\int_{\mathbb{R}} Q_{\varepsilon}(x) \psi(x) d x= & \frac{\ln \varepsilon}{\varepsilon} \int_{-\varepsilon}^{\varepsilon} \varkappa\left(\frac{x}{\varepsilon}\right) \psi(x) d x \\
& +q_{+} \int_{\varepsilon}^{a} \frac{\psi(x)}{x} d x+q_{-} \int_{-a}^{-\varepsilon} \frac{\psi(x)}{x} d x+\int_{|x|>a} Q(x) \psi(x) d x .
\end{aligned}
$$

If $\psi(0) \neq 0$, then all integrals on the right hand side are of the order $O(\ln \varepsilon)$ as $\varepsilon \rightarrow 0$, except for the last one. Indeed, we have

$$
\begin{aligned}
& \frac{\ln \varepsilon}{\varepsilon} \int_{-\varepsilon}^{\varepsilon} \varkappa\left(\frac{x}{\varepsilon}\right) \psi(x) d x-\psi(0) \ln \varepsilon \int_{\mathcal{I}} \varkappa d t=\ln \varepsilon \int_{-1}^{1} \varkappa(t)(\psi(\varepsilon t)-\psi(0)) d t \\
& \int_{\varepsilon}^{a} \frac{\psi(x)}{x} d x+\psi(0) \ln \varepsilon=\psi(0) \ln a+\int_{\varepsilon}^{a} \frac{\psi(x)-\psi(0)}{x} d x \\
& \int_{-a}^{-\varepsilon} \frac{\psi(x)}{x} d x-\psi(0) \ln \varepsilon=-\psi(0) \ln a+\int_{-a}^{-\varepsilon} \frac{\psi(x)-\psi(0)}{x} d x .
\end{aligned}
$$


The right-hand sides have finite limits as $\varepsilon \rightarrow 0$, since $\psi(x)-\psi(0)=O(x)$ as $x \rightarrow 0$. We obtain then

$$
\int_{\mathbb{R}} Q_{\varepsilon}(x) \psi(x) d x=\left(\int_{\mathcal{I}} \varkappa d t-q_{+}+q_{-}\right) \psi(0) \ln \varepsilon+f_{\varepsilon}(\psi),
$$

where $\left\{f_{\varepsilon}\right\}_{\varepsilon>0}$ is a sequence of continuous functionals which converges in $\mathcal{D}^{\prime}(\mathbb{R})$ as $\varepsilon \rightarrow 0$. Therefore sequence $Q_{\varepsilon}$ converges in the space of distributions if and only if $q_{+}-q_{-}=\int_{\mathcal{I}} \varkappa d t$.

Note that we have actually proved that condition (1.13) is necessary and sufficient for the convergence of functionals $Q_{\varepsilon}$ in Hölder space $C_{0}^{0, \gamma}(\mathbb{R}), \gamma \in(0,1)$. In fact, for any $\psi \in C_{0}^{0, \gamma}(\mathbb{R})$ we have $\psi(x)-\psi(0)=O\left(x^{\gamma}\right)$ as $x \rightarrow 0$, and this is sufficient for the convergence of $f_{\varepsilon}$. Therefore if $Q_{\varepsilon}$ converge in $\mathcal{D}^{\prime}(\mathbb{R})$, then the limit distribution belongs to $\mathcal{F}_{Q}$.

2.2. Proof of Theorem 2. The proof deals with the convergence of operators

$$
H_{\varepsilon}=-\frac{d^{2}}{d x^{2}}+Q_{\varepsilon}+\varepsilon^{-1} V\left(\varepsilon^{-1} \cdot\right),
$$

and so we have the partial case of Theorem 1 when $U=0$. First of all, note that the trivial potential $U=0$ possesses a zero-energy resonance with half-bound state $h_{0}=1$. Since $\theta=1,(1.7)$ and (1.8) become $\mu=\int_{\mathbb{R}} V d x=: \beta$ and

$$
q_{+}-q_{-}=\int_{\mathcal{I}} \varkappa d x
$$

respectively. Hence, if the last condition holds, then operators $H_{\varepsilon}$, given by (2.1), converge to operator $\mathcal{H}$ associated with non-trivial point interactions (1.14), according to Theorem 1. In this case we obtain a partial transparency of the potentials $Q_{\varepsilon}+\varepsilon^{-1} V\left(\varepsilon^{-1} \cdot\right)$ in the limit. Next, in view of Lemma 1 , the penetrability of these potentials is equivalent to the convergence of $Q_{\varepsilon}$ in the space of distributions.

2.3. Examples of Coulomb-like potentials. Following are some examples of potentials $Q_{\varepsilon}$, illustrating the penetrability and impenetrability of the Coulomblike potentials in the limit. Let us consider two regularizations of the classic Coulomb potential $Q(x)=-|x|^{-1}$ :

$$
Q_{0, \varepsilon}(x)=\left\{\begin{array}{cl}
-\frac{1}{|x|}, & \text { if }|x|>\varepsilon, \\
0, & \text { if }|x|<\varepsilon ;
\end{array} \quad Q_{1, \varepsilon}(x)=\left\{\begin{array}{cl}
-\frac{1}{|x|}, & \text { if }|x|>\varepsilon, \\
\varepsilon^{-1}|\ln \varepsilon|, & \text { if }|x|<\varepsilon
\end{array}\right.\right.
$$

(see Fig. 2). Both of the sequences $Q_{j, \varepsilon}$ converge to $-|x|^{-1}$ pointwise, but $Q_{1, \varepsilon}$ only converges in the sense of distributions. In the case of $Q_{1, \varepsilon}$, we have $q_{-}=1$, $q_{+}=-1$ and $\varkappa=-1$, and therefore condition (1.13) holds. In view of Theorem 2, potentials $Q_{0, \varepsilon}$ are asymptotically opaque; whereas $Q_{1, \varepsilon}$ are penetrable in the limit as $\varepsilon \rightarrow 0$. In other words, the transition probability $\left|T_{\varepsilon}(k)\right|^{2}$ calculated for $Q_{0, \varepsilon}$ tends to zero $\varepsilon \rightarrow 0$ for all $k$, but the corresponding probability for $Q_{1, \varepsilon}$ has a limit $|T(k)|^{2}$, which is a non-zero function of $k$. 

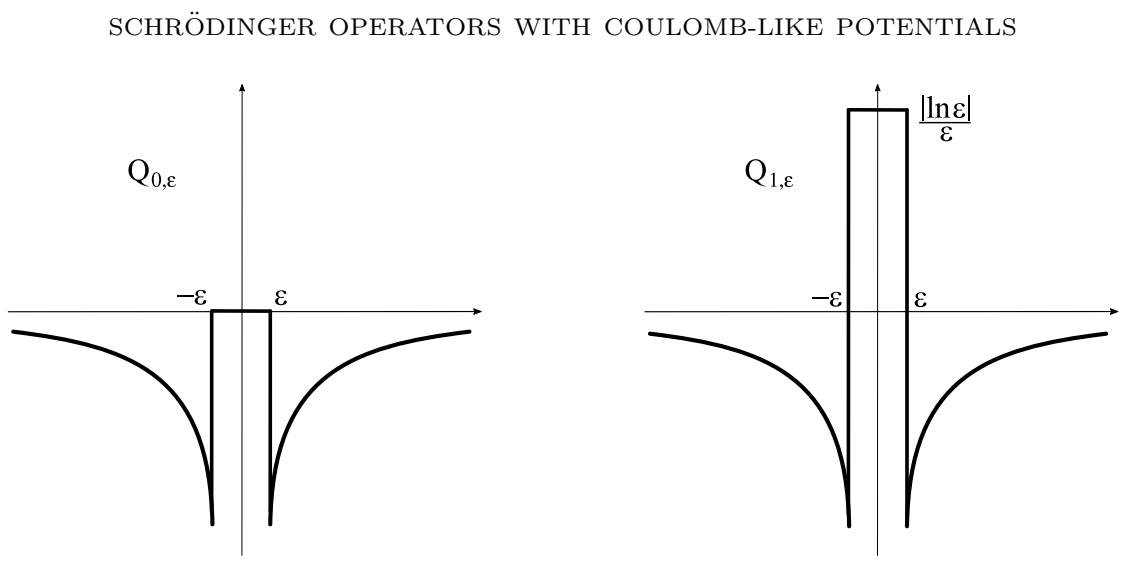

FiguRE 2. Impenetrable and penetrable regularizations of the even Coulomb potential

For the odd Coulomb potential $Q(x)=x^{-1}$, we can also provide two different regularizations, plotted in Fig. 3, as follows:

$$
Q_{2, \varepsilon}(x)=\left\{\begin{array}{cl}
\frac{1}{x}, & \text { if }|x|>\varepsilon \\
\varepsilon^{-1}|\ln \varepsilon|, & \text { if }|x|<\varepsilon
\end{array}, \quad Q_{3, \varepsilon}(x)=\left\{\begin{array}{cl}
\frac{1}{x}, & \text { if }|x|>\varepsilon, \\
\varepsilon^{-2}|\ln \varepsilon| x, & \text { if }|x|<\varepsilon
\end{array} .\right.\right.
$$

In this case, $q_{-}=q_{+}=1$ and so condition (1.13) holds for potentials $Q_{3, \varepsilon}$ only, when $\varkappa(t)=-t$. Therefore $Q_{3, \varepsilon}$ are penetrable in the limit as $\varepsilon \rightarrow 0$, unlike the potentials $Q_{2, \varepsilon}$, which are asymptotically opaque.

Many authors have regularized the Coulomb potentials by so-called truncated ones of the form

$$
R_{\varepsilon}(x)= \begin{cases}Q(x), & \text { if }|x|>\varepsilon, \\ a_{\varepsilon}(x), & \text { if }|x|<\varepsilon,\end{cases}
$$
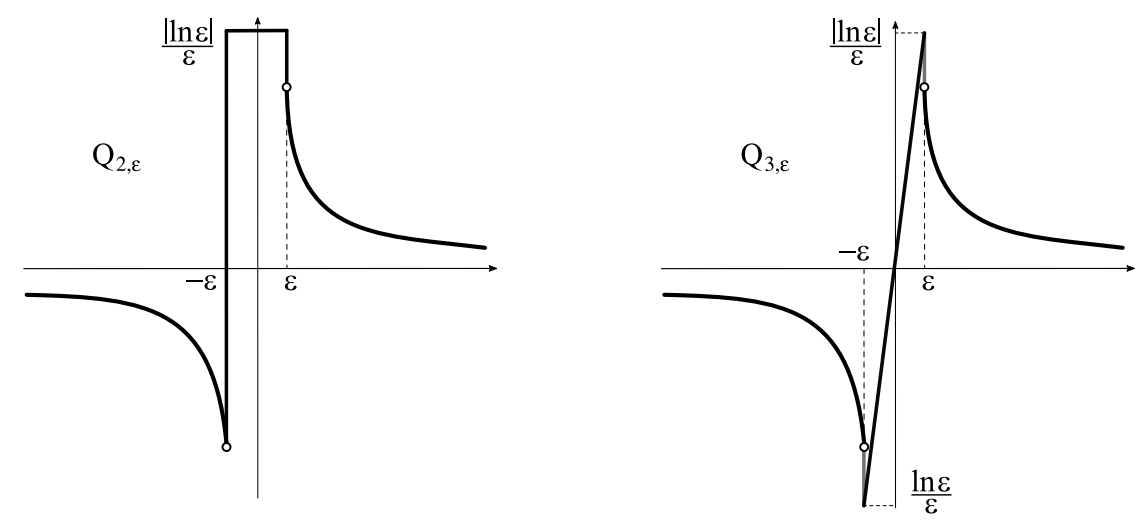

FiguRE 3. Impenetrable and penetrable regularizations of the odd Coulomb potential 
where $\left|a_{\varepsilon}\right| \leq c \varepsilon^{-1}$ (see Fig. 4). From asymptotical point of view, $R_{\varepsilon}$ can be regarded as potentials $Q_{\varepsilon}$ with $\varkappa=0$. It follows from the proof of Lemma 1 that $R_{\varepsilon}$ can converge in $\mathcal{D}^{\prime}(\mathbb{R})$ if and only if $q_{-}=q_{+}$, i.e., $Q(x)$ is the odd Coulomb potential near the origin. Moshinsky [8] was the first who noticed the penetrability of potential $\gamma / x$. On the other hand, a regularization of the even Coulomb potential $\gamma /|x|$ by the truncated potentials is always asymptotically opaque and leads to the Dirichlet condition in the limit $[1-3,7]$. The same assertion is also valid for modified Coulomb interactions having the form $M_{\varepsilon}(x)=-\frac{1}{|x|+\varepsilon}$; this potentials also diverge in $\mathcal{D}^{\prime}(\mathbb{R})$. Such regularizations were considered in $[1,2,4-6]$.
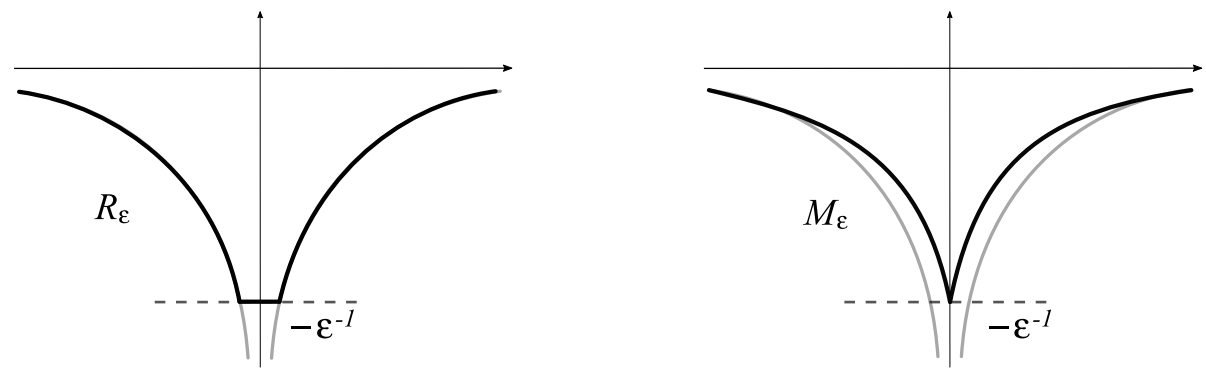

FiguRE 4. Truncated and modified potentials

It should be noted that the equivalence of penetrability in the limit and the convergence in the space of distributions for potentials $Q_{\varepsilon}+\varepsilon^{-1} V\left(\varepsilon^{-1} \cdot\right)$ is consistent with Kurasov's results $[12,14]$. Kurasov has interpreted the formal differential expression $-\frac{d^{2}}{d x^{2}}-\frac{\gamma}{x}$ in $\mathbb{R}$ as a map from some Hilbert space to the space of distributions. This operator has been defined in the principal value sense

$$
H=v \cdot p \cdot\left(-\frac{d^{2}}{d x^{2}}-\frac{\gamma}{x}\right)+\beta \delta(x)
$$

on the whole line. As shown in [14], $H$ is the self-adjoint operator that is defined by $H \phi=-\phi^{\prime \prime}-\gamma \phi / x$ on functions from $W_{2}^{2}(\mathbb{R} \backslash(-\varepsilon, \varepsilon))$ for every positive $\varepsilon>0$ and satisfying the boundary conditions

$$
\phi(+0)=\phi(-0), \quad b_{+}(\phi)-b_{-}(\phi)=\beta \phi(0),
$$

where $b_{ \pm}(\phi)=\lim _{x \rightarrow \pm 0}\left(\phi^{\prime}(x)-\gamma \phi( \pm 0) \ln |x|\right)$. Indeed, such considerations implicitly presupposed the existence of a regularization $Q_{\varepsilon}+\varepsilon^{-1} V\left(\varepsilon^{-1} \cdot\right)$ of the pseudopotential $-\mathcal{P} \frac{1}{x}+\beta \delta(x)$, which converges in $\mathcal{D}^{\prime}(\mathbb{R})$. These coupling conditions agree with (1.14), if $\gamma=q_{+}=q_{-}$.

Returning to the question of penetrability of the one-dimensional Coulomb potentials, it is probably worth considering that this question has no unambiguous answer. One should agree with the authors of [11] that mathematics alone cannot tell which boundary conditions for the wave function at the origin should be chosen to model a given experimental situation. 


\section{Proof of Theorem 1}

3.1. Formal construction of limit operator. Let us consider the equation

$$
-y^{\prime \prime}+(Q-\zeta) y=f, \quad x \in \mathbb{R} \backslash\{0\},
$$

for given $f \in L^{2}(\mathbb{R})$ and $\zeta \in \mathbb{C}$. In one-sided neighbourhoods of the origin the last equation becomes

$$
-y^{\prime \prime}+\left(\frac{q_{-}}{x}-\zeta\right) y=f, \quad x \in(-a, 0) ; \quad-y^{\prime \prime}+\left(\frac{q_{+}}{x}-\zeta\right) y=f, \quad x \in(0, a) .
$$

The following proposition was proved in [14].

Proposition 1. Let $y$ be a solution of (3.1) such that $y \in L^{2}(\mathbb{R})$. Then there exist the finite limits $y( \pm 0)=\lim _{x \rightarrow \pm 0} y(x)$ and

$$
y(x)=y( \pm 0)+O\left(|x|^{1 / 2}\right) \quad \text { as } x \rightarrow \pm 0 .
$$

For the derivative of the solution we have asymptotics

$$
y^{\prime}(x)=q_{ \pm} y( \pm 0) \ln |x|+b_{ \pm}(y)+o(1) \quad \text { as } x \rightarrow \pm 0,
$$

where $b_{-}$and $b_{+}$are some constants depending on $y$.

We will use this proposition for some formal considerations. To proof the norm resolvent convergence of $H_{\varepsilon}$ we do really need more subtle estimates of the remainder terms; a stronger version of these asymptotics is presented in Lemma 2. Set $y_{\varepsilon}=\left(H_{\varepsilon}-\zeta\right)^{-1} f$ for $f \in L^{2}(\mathbb{R})$ and $\zeta \in \mathbb{C} \backslash \mathbb{R}$. We look for the formal asymptotics of $y_{\varepsilon}$, as $\varepsilon \rightarrow 0$, having the form

$$
y_{\varepsilon}(x) \sim \begin{cases}u(x), & \text { if }|x|>\varepsilon \\ v_{0}\left(\frac{x}{\varepsilon}\right)+v_{1}\left(\frac{x}{\varepsilon}\right) \varepsilon \ln \varepsilon+v_{2}\left(\frac{x}{\varepsilon}\right) \varepsilon, & \text { if }|x|<\varepsilon .\end{cases}
$$

We also assume that the coupling conditions

$$
\left[y_{\varepsilon}\right]_{ \pm \varepsilon}=0, \quad\left[y_{\varepsilon}^{\prime}\right]_{ \pm \varepsilon}=0
$$

hold, where $[\cdot]_{x}$ is the jump of a function at point $x$. Function $y_{\varepsilon}$ is a unique solution of equation

$$
-y_{\varepsilon}^{\prime \prime}+\left(Q_{\varepsilon}(x)+\varepsilon^{-2} U\left(\varepsilon^{-1} x\right)+\varepsilon^{-1} V\left(\varepsilon^{-1} x\right)\right) y_{\varepsilon}=\zeta y_{\varepsilon}+f
$$

belonging to the domain of $H_{\varepsilon}$. Since the interval on which the $\left(\alpha \delta^{\prime}+\beta \delta\right)$-like perturbation is localized shrinks to a point, $u$ must solve the equation

$$
-u^{\prime \prime}+Q u=\zeta u+f \quad \text { in } \mathbb{R} \backslash\{0\} .
$$

This solution can not be uniquely determined without additional conditions at the origin. One naturally expects that these conditions depend on the perturbation.

Suppose that $|x|<\varepsilon<a$. Then we can as follows rewrite equation (3.4) in the terms of new variable $t=x / \varepsilon$. If we set $v^{\varepsilon}(t)=y_{\varepsilon}(\varepsilon t)$, then

$$
-\frac{d^{2} v^{\varepsilon}}{d t^{2}}+(U(t)+\varepsilon \ln \varepsilon \varkappa(t)+\varepsilon V(t)) v^{\varepsilon}=\varepsilon^{2} \zeta v^{\varepsilon}+\varepsilon^{2} f, \quad t \in \mathcal{I} .
$$


Furthermore, in view of Proposition 1, matching conditions (3.3) imply

$$
\begin{aligned}
v_{0}( \pm 1)+O(\varepsilon \ln \varepsilon) & =u( \pm 0)+O\left(\varepsilon^{1 / 2}\right), \\
\varepsilon^{-1} v_{0}^{\prime}( \pm 1)+v_{1}^{\prime}( \pm 1) \ln \varepsilon+v_{2}^{\prime}( \pm 1) & =q_{ \pm} u( \pm 0) \ln \varepsilon+b_{ \pm}(u)+o(1) .
\end{aligned}
$$

In particular, we have

$$
v_{0}( \pm 1)=u( \pm 0), \quad v_{0}^{\prime}( \pm 1)=0, \quad v_{1}^{\prime}( \pm 1)=q_{ \pm} u( \pm 0), \quad v_{2}^{\prime}( \pm 1)=b_{ \pm}(u) .
$$

Substituting (3.2) for $|x|<\varepsilon$ into (3.5) and applying (3.6) yield

$$
\begin{array}{ll}
-v_{0}^{\prime \prime}+U v_{0}=0, \quad t \in \mathcal{I}, \quad & v_{0}^{\prime}(-1)=0, \quad v_{0}^{\prime}(1)=0 ; \\
-v_{1}^{\prime \prime}+U v_{1}=-\varkappa v_{0}, \quad t \in \mathcal{I}, & v_{1}^{\prime}(-1)=q_{-} u(-0), \quad v_{1}^{\prime}(1)=q_{+} u(+0) ; \\
-v_{2}^{\prime \prime}+U v_{2}=-V v_{0}, \quad t \in \mathcal{I}, & v_{2}^{\prime}(-1)=b_{-}(u), \quad v_{2}^{\prime}(1)=b_{+}(u) .
\end{array}
$$

Let us first suppose that potential $U$ is resonant. Since the supports of $U$ is contained in $\mathcal{I}$, a half-bound state $h$ is constant outside $\mathcal{I}$ and its restriction to $\mathcal{I}$ is a non-trivial solution of the boundary value problem

$$
-h^{\prime \prime}+U h=0, \quad t \in \mathcal{I}, \quad h^{\prime}(-1)=0, \quad h^{\prime}(1)=0 .
$$

Moreover $h( \pm \infty)=h( \pm 1)$ and hence $h_{0}(-1)=1$ and $h_{0}(1)=\theta$. Then the equation in (3.7) has a one-parameter family of solutions $v_{0}=c h_{0}$. But owing to $v_{0}(-1)=u(-0)$, we have

$$
v_{0}=u(-0) h_{0} .
$$

Hence $v_{0}(1)=u(-0) h_{0}(1)=\theta u(-0)$. On the other hand, $v_{0}(1)=u(+0)$ by $(3.6)$. From this we deduce

$$
u(+0)=\theta u(-0) .
$$

Next, problem (3.8) is solvable if and only if

$$
\theta q_{+} u(+0)-q_{-} u(-0)=u(-0) \int_{\mathcal{I}} x h_{0}^{2} d x,
$$

because the corresponding homogeneous problem possesses non-trivial solutions. The last condition can be easy obtained by multiplying the equation in (3.8) by $h_{0}$ and integrating by parts. Combining (3.12) and (3.13) gives us

$$
\left\{\begin{array}{c}
u(+0)-\theta u(-0)=0, \\
\theta q_{+} u(+0)-\left(q_{-}+\int_{\mathcal{I}} \varkappa h_{0}^{2} d x\right) u(-0)=0 .
\end{array}\right.
$$

The linear system admits a nonzero solution $(u(-0), u(+0))$ if and only if

$$
\theta^{2} q_{+}-q_{-}=\int_{\mathcal{I}} \varkappa h_{0}^{2} d x
$$

If (3.13) holds, then (3.8) has a one-parameter family of solutions $v_{1}=v_{1}^{*}+c_{1} h_{0}$. Let us fix $v_{1}$ such that $v_{1}(-1)=0$; this is possible, because $h_{0}(-1) \neq 0$.

We at last turn to problem (3.9). Multiplying the equation in (3.9) by halfbound state $h_{0}$ and integrating by parts, we can similarly compute the solvability condition

$$
\theta b_{+}(u)-b_{-}(u)=\int_{\mathcal{I}} V v_{0} h_{0} d t .
$$


We can choose $v_{2}$ to satisfy $v_{2}(-1)=0$. Recalling now (3.11), we can rewrite (3.16) in the form

$$
\theta b_{+}(u)-b_{-}(u)=u(-0) \int_{\mathcal{I}} V h_{0}^{2} d t
$$

Therefore if potential $U$ is resonant and (3.15) holds, then the leading term $u$ of asymptotics (3.2) must solve the problem

$$
\begin{gathered}
-u^{\prime \prime}+Q u=\zeta u+f \quad \text { in } \mathbb{R} \backslash\{0\}, \\
u(+0)-\theta u(-0)=0, \quad \theta b_{+}(u)-b_{-}(u)=\mu u(-0),
\end{gathered}
$$

where $\mu$ is given by (1.7). The coupling conditions at the origin agree with (1.9) in view of Remark 2.

In the case when either $U$ has no zero-energy resonance or else $U$ is resonant, but $(3.15)$ does not hold, both the values $u(-0)$ and $u(+0)$ equal zero. Indeed, if $U$ is not resonant, then problem (3.7) has only trivial solution $v_{0}$ and then the first condition in (3.6) implies $u(0)=0$. On the other hand, if $U$ is resonant, but (3.15) does not hold, then system (3.14) has a unique solution $u(-0)=u(+0)=0$. Hence $u$ should be a solution of the problem

$$
-u^{\prime \prime}+Q u=\zeta u+f \quad \text { in } \mathbb{R} \backslash\{0\}, \quad u(0)=0 .
$$

3.2. Improvement of asymptotics. We will again focus our attention on the case of resonant potential $U$. Our aim is to construct an element $u_{\varepsilon} \in \operatorname{dom} H_{\varepsilon}$ that approximates $y_{\varepsilon}=\left(H_{\varepsilon}-\zeta\right)^{-1} f$.

From now on, $W_{2}^{k}(\Omega)$ and $W_{2}^{k, l o c}(\Omega)$ stand for the Sobolev spaces and $\|f\|$ stands for $L^{2}(\mathbb{R})$-norm of a function $f$. To obtain the uniform approximation of $y_{\varepsilon}$ in $L^{2}(\mathbb{R})$ with respect to $f$, we will refine asymptotics (3.2). Let $z_{\varepsilon}$ be a solution of the Cauchy problem

$$
-z^{\prime \prime}+U(t) z=f(\varepsilon t), \quad z(-1)=0, \quad z^{\prime}(-1)=0 .
$$

We introduce the function

$$
w_{\varepsilon}(x)= \begin{cases}u(x), & \text { if }|x|>\varepsilon, \\ v_{0}\left(\frac{x}{\varepsilon}\right)+v_{1}\left(\frac{x}{\varepsilon}\right) \varepsilon \ln \varepsilon+v_{2}\left(\frac{x}{\varepsilon}\right) \varepsilon+\varepsilon^{2} z_{\varepsilon}\left(\frac{x}{\varepsilon}\right), & \text { if }|x|<\varepsilon .\end{cases}
$$

Note that $w_{\varepsilon}$ is not in general smooth enough to belong to the domain of $H_{\varepsilon}$; by construction, approximation $w_{\varepsilon}$ belongs to $W_{2, l o c}^{2}(\mathbb{R} \backslash\{-\varepsilon, \varepsilon\})$ and has jump discontinuities at the points $x= \pm \varepsilon$. We will show that the jumps of $w_{\varepsilon}$ and its first derivative are small enough uniformly on $f$, and therefore there exists a corrector $\rho_{\varepsilon}$ with the infinitesimal $L^{2}$-norm, as $\varepsilon \rightarrow 0$, such that $w_{\varepsilon}+\rho_{\varepsilon} \in \operatorname{dom} H_{\varepsilon}$.

We introduce two cut-functions $\xi$ and $\eta$ that are smooth outside the origin and have compact supports contained in $\left[0, \frac{1}{2} a\right]$, where $a$ is the same as in (1.2). In addition, $\xi(+0)=1, \xi^{\prime}(+0)=0, \eta(+0)=0$ and $\eta^{\prime}(+0)=1$. Let us set

$$
\rho_{\varepsilon}(x)=\left[w_{\varepsilon}\right]_{-\varepsilon} \xi(-x-\varepsilon)-\left[w_{\varepsilon}^{\prime}\right]_{-\varepsilon} \eta(-x-\varepsilon)-\left[w_{\varepsilon}\right]_{\varepsilon} \xi(x-\varepsilon)-\left[w_{\varepsilon}^{\prime}\right]_{\varepsilon} \eta(x-\varepsilon) .
$$

It is easy to check that $\left[\rho_{\varepsilon}^{(k)}\right]_{ \pm \varepsilon}=-\left[w_{\varepsilon}^{(k)}\right]_{ \pm \varepsilon}$ for $k=0,1$. Moreover, $\rho_{\varepsilon}(x)=0$ for $x \in(-\varepsilon, \varepsilon)$. From this we conclude that $w_{\varepsilon}+\rho_{\varepsilon} \in W_{2, l o c}^{2}(\mathbb{R})$, hence that $w_{\varepsilon}+\rho_{\varepsilon} \in \operatorname{dom} H_{\varepsilon}$. 
3.3. Some uniform bounds. Recall that in Subsection 3.1 we have actually derived $u=(\mathcal{H}-\zeta)^{-1} f$. Hence $u \in W_{2, l o c}^{2}(\mathbb{R} \backslash(-b, b))$ for any $b>0$. By the Sobolev imbedding theorems, function $u$ is continuously differentiable on $\mathbb{R} \backslash\{0\}$. In addition, the estimates hold

$$
\|u\| \leq c_{1}\|f\|, \quad\|u\|_{C^{1}(K)} \leq c_{2}(K)\|f\|
$$

for any compact set $K$ that does not contain the origin.

Lemma 2. The following estimates

$$
\begin{gathered}
|u(x)-u( \pm 0)| \leq C_{1}\|f\||x \ln | x \|, \\
\left|u^{\prime}(x)-q_{ \pm} u( \pm 0) \ln \right| x\left|-b_{ \pm}(u)\right| \leq C_{2}\|f\||x|^{1 / 2}
\end{gathered}
$$

hold as $x \rightarrow \pm 0$, where $b_{ \pm}$are linear bounded functionals on $\operatorname{dom} \mathcal{H}$. In addition,

$$
\left|b_{ \pm}(u)\right| \leq C_{3}\|f\|
$$

The constants $C_{k}$ do not depend on $f$.

Proof. We will prove (3.24)-(3.26) on the positive half-line only. For the case $x \rightarrow-0$ the proof is similar. In view of (1.2), we have

$$
u^{\prime \prime}=\frac{q_{+}}{x} u-\zeta u-f
$$

for $x \in(0, a)$. Temporarily write $f_{\zeta}=\zeta u+f$. Consequently

$$
\begin{aligned}
& u^{\prime}(x)=-q_{+} \int_{x}^{a} \frac{u(s)}{s} d s+\int_{x}^{a} f_{\zeta}(s) d s+u^{\prime}(a), \\
& u(x)=q_{+} \int_{x}^{a} \frac{s-x}{s} u(s) d s-\int_{x}^{a}(s-x) f_{\zeta}(s) d s+u(a)+u^{\prime}(a)(x-a) .
\end{aligned}
$$

From this we see in particular that there exists the finite limit value

$$
u(+0)=q_{+} \int_{0}^{a} u(s) d s+x \int_{0}^{a} f_{\zeta}(s) d s+u(a)-a u^{\prime}(a)
$$

not only for an element of dom $\mathcal{H}$, but for any $L^{2}\left(\mathbb{R}_{+}\right)$-solution of (3.27). In fact, the most singular (as $x \rightarrow+0$ ) integral

$$
\int_{x}^{a} \frac{s-x}{s} u(s) d s
$$

converges to $\int_{0}^{a} u(s) d s$ by Lebesgue's dominated convergence theorem, because

$$
\left|\frac{s-x}{s} \chi_{(x, a)}(s)\right| \leq 1 \quad \text { for } s \in(0, a) .
$$

Here $\chi_{(x, a)}$ is the characteristic function of interval $(x, a)$. Combining $(3.30)$ and the second inequality in (3.23), we discover

$$
\|u\|_{C^{0}([0, a])} \leq c\|f\|
$$


Subtracting (3.30) from (3.29), we can represent the difference as

$$
\begin{aligned}
u(x)-u(+0)=-q_{+} x \int_{x}^{a} & \frac{u(s)}{s} d s \\
& +\int_{0}^{x}\left(s f_{\zeta}(s)-q_{+} u(s)\right) d s+x \int_{x}^{a} f_{\zeta}(s) d s+u^{\prime}(a) x .
\end{aligned}
$$

Since

$$
\int_{x}^{a} \frac{u(s)}{s} d s=u(+0) x(\ln a-\ln x)+\int_{x}^{a} \frac{u(s)-u(+0)}{s} d s,
$$

we finally have

$$
\begin{aligned}
u(x)-u(+0)=q_{+} & u(+0) x(\ln x-\ln a)+\int_{0}^{x}\left(s f_{\zeta}(s)-q_{+} u(s)\right) d s \\
& +x \int_{x}^{a} f_{\zeta}(s) d s+u^{\prime}(a) x-q_{+} x \int_{x}^{a} \frac{u(s)-u(+0)}{s} d s .
\end{aligned}
$$

Hence

$$
|u(x)-u(+0)| \leq c_{1}\|f\| x|\ln x|+\left|q_{+}\right| x \int_{x}^{a} \frac{|u(s)-u(+0)|}{s} d s,
$$

where we employed (3.23) and (3.31) to obtain the estimates

$$
\begin{aligned}
|u(+0)| & +\left|u^{\prime}(a)\right| \leq c_{2}\|f\|, \quad\left\|f_{\zeta}\right\|=\|\zeta u+f\| \leq c_{3}\|f\|, \\
& \left|\int_{0}^{x} u(s) d s\right| \leq x \sup _{s \in(0, x)}|u(s)| \leq c_{4} x\|f\|, \\
& \left|\int_{0}^{x} s f_{\zeta}(s) d s\right| \leq x \int_{0}^{x}\left|f_{\zeta}(s)\right| d s \leq x^{3 / 2}\|f\| .
\end{aligned}
$$

Consequently Gronwall's inequality implies

$$
|u(x)-u(+0)| \leq c_{1}\|f\| e^{\left|q_{+}\right| x(\ln a-\ln x)} x|\ln x| \leq C_{1}\|f\| x|\ln x|,
$$

as $x \rightarrow+0$, which establishes (3.24). Applying (3.32) to (3.28), we find

$$
\begin{aligned}
u^{\prime}(x)=q_{+} u(+0)(\ln x & -\ln a)-q_{+} \int_{x}^{a} \frac{u(s)-u(+0)}{s} d s \\
& +\int_{x}^{a} f_{\zeta}(s) d s+u^{\prime}(a)=q_{+} u(+0) \ln x+b_{+}(u)+r(x, u),
\end{aligned}
$$

where

$$
\begin{aligned}
& b_{+}(u)=u^{\prime}(a)-q_{+} u(+0) \ln a+\int_{0}^{a} f_{\zeta}(s) d s-q_{+} \int_{0}^{a} \frac{u(s)-u(+0)}{s} d s \\
& r(x, u)=q_{+} \int_{0}^{x} \frac{u(s)-u(+0)}{s} d s-\int_{0}^{x} f_{\zeta}(s) d s .
\end{aligned}
$$


Thus formulas (3.23), (3.24) and (3.31) provide the bounds

$$
\begin{aligned}
|r(x, u)| & \leq\left|q_{+}\right| \int_{0}^{x} \frac{|u(s)-u(+0)|}{s} d s+|\zeta| \int_{0}^{x}|u| d s+\int_{0}^{x}|f| d s \\
& \leq C_{1}\|f\| \int_{0}^{x}|\ln s| d s+c_{4}(\|u\|+\|f\|) x^{1 / 2} \leq C_{2}\|f\| x^{1 / 2}, \\
\left|b_{+}(u)\right| & \leq c_{5}\left(\left|u^{\prime}(a)\right|+|u(+0)|\right)+c_{6}\left\|f_{\zeta}\right\| \\
& +\left|q_{+}\right| \int_{0}^{a} \frac{|u(s)-u(+0)|}{s} d s \leq c_{7}\|f\|+c_{8}\|f\| \int_{0}^{a}|\ln s| d s \leq C_{3}\|f\|,
\end{aligned}
$$

which establishes (3.25) and (3.26).

By construction functions $v_{k}$ in $(3.2)$ belong to $W_{2}^{2}(\mathcal{I})$. We will show that their $W_{2}^{2}$-norms can be estimated by the $L^{2}$-norm of $f$.

Lemma 3. Assume that $v_{0}, v_{1}$ and $v_{2}$ are solunions of (3.7), (3.8) and (3.9) respectively. Suppose that these solutions are chosen so that $v_{0}(-1)=u(-0)$, $v_{1}(-1)=0$ and $v_{2}(-1)=0$. Then

$$
\left\|v_{k}\right\|_{W_{2}^{2}(\mathcal{I})} \leq C_{1}\|f\|
$$

for all $f \in L^{2}(\mathbb{R})$ and $k=0,1,2$, the constant $C_{1}$ being independent of $f$.

Let $z_{\varepsilon}$ be the solution of $(3.20)$. Then $z_{\varepsilon}$ also belongs to $W_{2}^{2}(\mathcal{I})$, with the estimate

$$
\left\|z_{\varepsilon}\right\|_{W_{2}^{2}(\mathcal{I})} \leq C_{2} \varepsilon^{-1 / 2}\|f\|
$$

where $C_{2}$ does not depend of $f$ and $\varepsilon$.

Proof. It is evident from (3.11) and Lemma 2 that $\left\|v_{0}\right\|_{W_{2}^{2}(\mathcal{I})} \leq c|u(-0)| \leq C_{1}\|f\|$. To prove this estimate for $v_{1}$ and $v_{2}$, we construct below representations for the desired solutions. Let $\omega$ be a solution of the Cauchy problem

$$
-\omega^{\prime \prime}+U \omega=-\varkappa h_{0}, \quad t \in \mathcal{I}, \quad \omega(-1)=0, \quad \omega^{\prime}(-1)=q_{-} .
$$

We set $v_{1}=u(-0) \omega$. This function solves the equation in (3.8) and $v_{1}^{\prime}(-1)=$ $q_{-} u(-0)$. The boundary condition at $t=1$ also holds, because multiplying the equation for $\omega$ by half-bound state $h_{0}$ and integrating by parts twice yield

$$
\theta \omega^{\prime}(1)=q_{-}+\int_{\mathcal{I}} \varkappa h_{0}^{2} d x
$$

From this we have

$$
v_{1}^{\prime}(1)=u(-0) \omega^{\prime}(1)=\theta^{-1} u(-0)\left(q_{-}+\int_{\mathcal{I}} \varkappa h_{0}^{2} d x\right)=q_{+} \theta u(-0)=q_{+} u(+0),
$$

by (1.8). Next, solution $v_{2}$ of (3.9) can be written as

$$
v_{2}(t)=b_{-}(u) \omega(t)+u(-0) \Omega(t)
$$

where $\Omega$ solves the problem

$$
-\Omega^{\prime \prime}+U \Omega=-V h_{0}, \quad t \in \mathcal{I}, \quad \Omega(-1)=0, \quad \Omega^{\prime}(-1)=0 .
$$

Note that $\Omega^{\prime}(1)=\theta^{-1} \int_{\mathbb{R}} V h_{0}^{2} d t$. This equality can be obtained by multiplying equation $-y^{\prime \prime}+U y=-V h_{0}$ by half-bound state $h_{0}$ and integrating by parts. 
So we have $v_{2}(-1)=b_{-}(u) \omega(-1)+u(-0) \Omega(-1)=0, v_{2}^{\prime}(-1)=b_{-}(u) \omega^{\prime}(-1)+$ $u(-0) \Omega^{\prime}(-1)=b_{-}(u)$ and

$$
v_{2}^{\prime}(1)=b_{-}(u) \omega^{\prime}(1)+u(-0) \Omega^{\prime}(1)=\theta^{-1}\left(b_{-}(u)+u(-0) \int_{\mathcal{I}} V h_{0}^{2} d t\right)=b_{+}(u)
$$

in view of coupling condition (3.17). Hence $v_{2}$ of the form (3.37) is a solution of (3.9) such that $v_{2}(-1)=0$. Estimate (3.35) for $k=1,2$ follows from the explicit form of $v_{1}, v_{2}$, bounds (3.23) and Lemma 2.

Since $U \in L^{\infty}(\mathbb{R})$, solution $z_{\varepsilon}$ of the Cauchy problem satisfies

$$
\left\|z_{\varepsilon}\right\|_{W_{2}^{2}(\mathcal{I})} \leq c_{1}\|f(\varepsilon \cdot)\|_{L^{2}(\mathcal{I})} .
$$

We also have

$$
\int_{-1}^{1}|f(\varepsilon t)|^{2} d t \leq c_{2} \varepsilon^{-1} \int_{-\varepsilon}^{\varepsilon}|f(\tau)|^{2} d \tau \leq c_{3} \varepsilon^{-1}\|f\|^{2} .
$$

Therefore (3.36) follows from the last bound.

Lemma 4. Assume that function $\rho_{\varepsilon}$ is given by (3.22). There exist constants $C_{1}$ and $C_{2}$ being independent of $f$ such that

$$
\begin{gathered}
\sup _{|x|>\varepsilon}\left(\left|\rho_{\varepsilon}(x)\right|+\left|\rho_{\varepsilon}^{\prime \prime}(x)\right|\right) \leq C_{1} \varepsilon^{1 / 2}\|f\|, \\
\left\|Q \rho_{\varepsilon}\right\| \leq C_{2} \varepsilon^{1 / 4}\|f\| .
\end{gathered}
$$

Proof. To prove (3.38) it suffices to show

$$
\left|\left[w_{\varepsilon}\right]_{-\varepsilon}\right|+\left|\left[w_{\varepsilon}\right]_{\varepsilon}\right|+\left|\left[w_{\varepsilon}^{\prime}\right]_{-\varepsilon}\right|+\left|\left[w_{\varepsilon}^{\prime}\right]_{\varepsilon}\right| \leq c \varepsilon^{1 / 2}\|f\|,
$$

since functions $\xi$ and $\eta$ in (3.22) are smooth and bounded together with all their derivatives, if $|x|>\varepsilon$. Combining Lemmas 2, 3 and the continuity of embedding $W_{2}^{2}(\mathcal{I}) \subset C^{1}(\mathcal{I})$, we conclude that

$$
\begin{aligned}
& \left|\left[w_{\varepsilon}\right]_{-\varepsilon}\right|=\left|v_{0}(-1)-u(-\varepsilon)\right|=|u(-0)-u(-\varepsilon)| \leq c_{1}\|f\| \varepsilon|\ln \varepsilon| \\
& \left|\left[w_{\varepsilon}^{\prime}\right]_{-\varepsilon}\right|=\left|u^{\prime}(-\varepsilon)-q_{-} u(-0) \ln \varepsilon-b_{-}(u)\right| \leq c_{2}\|f\| \varepsilon^{1 / 2} \\
& \left|\left[w_{\varepsilon}\right]_{\varepsilon}\right|=\left|u(\varepsilon)-u(+0)-v_{1}(1) \varepsilon \ln \varepsilon-v_{2}(1) \varepsilon-z_{\varepsilon}(\varepsilon) \varepsilon^{2}\right| \leq c_{3}\|f\| \varepsilon|\ln \varepsilon|, \\
& \left|\left[w_{\varepsilon}^{\prime}\right]_{\varepsilon}\right|=\left|u^{\prime}(\varepsilon)-q_{+} u(+0) \ln \varepsilon-b_{+}(u)-z_{\varepsilon}^{\prime}(\varepsilon) \varepsilon\right| \leq c_{4}\|f\| \varepsilon^{1 / 2}
\end{aligned}
$$

which establishes (3.38).

Next, let us fix $\gamma \in\left(0, \frac{1}{2}\right)$. Since $|\eta(x)| \leq c|x|$ as $|x| \rightarrow 0$, we have

$$
\begin{aligned}
\sup _{\varepsilon<|x|<\varepsilon^{\gamma}}\left|\rho_{\varepsilon}(x)\right| \leq c_{5}\left(\left|\left[w_{\varepsilon}\right]_{-\varepsilon}\right|\right. & \left.+\left|\left[w_{\varepsilon}\right]_{\varepsilon}\right|+\left(\left|\left[w_{\varepsilon}^{\prime}\right]_{-\varepsilon}\right|+\left|\left[w_{\varepsilon}^{\prime}\right]_{\varepsilon}\right|\right) \varepsilon^{\gamma}\right) \\
& \leq c_{6}\left(\varepsilon|\ln \varepsilon|+\varepsilon^{\gamma+1 / 2}\right)\|f\| \leq c_{7} \varepsilon^{\gamma+1 / 2}\|f\|
\end{aligned}
$$


for $\varepsilon<|x|<\varepsilon^{\gamma}$. Recall that $\rho_{\varepsilon}(x)=0$ for $|x|<\varepsilon$ and $|x|>a$, provided $\varepsilon$ is small enough. Then utilizing estimates (3.38) and (3.40), we obtain the bound

$$
\begin{aligned}
\left\|Q \rho_{\varepsilon}\right\|^{2} & =\int_{\varepsilon<|x|<a} Q^{2}\left|\rho_{\varepsilon}\right|^{2} d x \leq \max \left\{\left|q_{-}\right|,\left|q_{+}\right|\right\} \int_{\varepsilon<|x|<a} x^{-2}\left|\rho_{\varepsilon}\right|^{2} d x \\
& \leq c_{8}\left(\int_{\varepsilon<|x|<\varepsilon^{\gamma}} x^{-2}\left|\rho_{\varepsilon}\right|^{2} d x+\int_{\varepsilon^{\gamma}<|x|<a} x^{-2}\left|\rho_{\varepsilon}\right|^{2} d x\right) \\
& \leq c_{8} \sup _{\varepsilon<|x|<\varepsilon^{\gamma}}\left|\rho_{\varepsilon}(x)\right|^{2} \int_{\varepsilon<|x|<\varepsilon^{\gamma}} x^{-2} d x+c_{8} \sup _{\varepsilon^{\gamma}<|x|<a} x^{-2}\left|\rho_{\varepsilon}(x)\right|^{2} \int_{\varepsilon^{\gamma}<|x|<a} d x \\
& \leq c_{9} \varepsilon^{2 \gamma+1}\|f\| \int_{\varepsilon<|x|<\varepsilon^{\gamma}} x^{-2} d x+c_{10} \varepsilon^{1-2 \gamma}\|f\| \leq c_{11}\left(\varepsilon^{2 \gamma}+\varepsilon^{1-2 \gamma}\right)\|f\| .
\end{aligned}
$$

Assertion (3.39) follows from this inequality, provided $\gamma=1 / 4$.

3.4. End of the proof. We showed above that $u_{\varepsilon}=w_{\varepsilon}+\rho_{\varepsilon}$ belongs to the domain of $H_{\varepsilon}$. We will now prove that $u_{\varepsilon}$ solves the equation

$$
\left(H_{\varepsilon}-\zeta\right) u_{\varepsilon}=f+g_{\varepsilon},
$$

in which remainder term $g_{\varepsilon}$ is small in $L_{2}$-norm uniformly with respect to $f$. Let us compute $g_{\varepsilon}$. If $|x|>\varepsilon$, then we have

$$
g_{\varepsilon}(x)=\left(-\frac{d^{2}}{d x^{2}}+Q(x)-\zeta\right)\left(u(x)+\rho_{\varepsilon}(x)\right)-f(x)=-\rho_{\varepsilon}^{\prime \prime}(x)+(Q(x)-\zeta) \rho_{\varepsilon}(x),
$$

by (3.18). If $|x|<\varepsilon$, then

$$
\begin{aligned}
g_{\varepsilon}(x) & =-\frac{d^{2}}{d x^{2}} u_{\varepsilon}\left(\frac{x}{\varepsilon}\right)+\left(\varepsilon^{-2} U\left(\frac{x}{\varepsilon}\right)+\varepsilon^{-1} \ln \varepsilon \varkappa\left(\frac{x}{\varepsilon}\right)+\varepsilon^{-1} V\left(\frac{x}{\varepsilon}\right)-\zeta\right) u_{\varepsilon}\left(\frac{x}{\varepsilon}\right)-f(x) \\
& =\varepsilon^{-2}\left(-v_{0}^{\prime \prime}\left(\frac{x}{\varepsilon}\right)+U\left(\frac{x}{\varepsilon}\right) v_{0}\left(\frac{x}{\varepsilon}\right)\right) \\
& +\varepsilon^{-1} \ln \varepsilon\left(-v_{1}^{\prime \prime}\left(\frac{x}{\varepsilon}\right)+U\left(\frac{x}{\varepsilon}\right) v_{1}\left(\frac{x}{\varepsilon}\right)+\varkappa\left(\frac{x}{\varepsilon}\right) v_{0}\left(\frac{x}{\varepsilon}\right)\right) \\
& +\varepsilon^{-1}\left(-v_{2}^{\prime \prime}\left(\frac{x}{\varepsilon}\right)+U\left(\frac{x}{\varepsilon}\right) v_{2}+V\left(\frac{x}{\varepsilon}\right) v_{0}\left(\frac{x}{\varepsilon}\right)\right) \\
& -z_{\varepsilon}^{\prime \prime}\left(\frac{x}{\varepsilon}\right)+U\left(\frac{x}{\varepsilon}\right) z_{\varepsilon}\left(\frac{x}{\varepsilon}\right)-f(x) \\
& +\ln \varepsilon \varkappa\left(\frac{x}{\varepsilon}\right)\left(v_{1}\left(\frac{x}{\varepsilon}\right) \ln \varepsilon+v_{2}\left(\frac{x}{\varepsilon}\right)+\varepsilon z_{\varepsilon}\left(\frac{x}{\varepsilon}\right)\right) \\
& +V\left(\frac{x}{\varepsilon}\right)\left(v_{1}\left(\frac{x}{\varepsilon}\right) \ln \varepsilon+v_{2}\left(\frac{x}{\varepsilon}\right)+\varepsilon z_{\varepsilon}\left(\frac{x}{\varepsilon}\right)\right)-\zeta u_{\varepsilon}\left(\frac{x}{\varepsilon}\right) \\
& =\left(\varkappa\left(\frac{x}{\varepsilon}\right) \ln \varepsilon+V\left(\frac{x}{\varepsilon}\right)\right)\left(v_{1}\left(\frac{x}{\varepsilon}\right) \ln \varepsilon+v_{2}\left(\frac{x}{\varepsilon}\right)+z_{\varepsilon}\left(\frac{x}{\varepsilon}\right) \varepsilon\right)-\zeta u_{\varepsilon}\left(\frac{x}{\varepsilon}\right)
\end{aligned}
$$

by (3.7)-(3.9) and (3.20). Hence we have

$$
\begin{array}{r}
\left\|g_{\varepsilon}\right\| \leq\left\|\rho_{\varepsilon}^{\prime \prime}+\zeta \rho_{\varepsilon}\right\|+\left\|Q \rho_{\varepsilon}\right\|+\sup _{t \in \mathcal{I}}(|U(t) \| \ln \varepsilon|+|V(t)|) \\
\times\left\|v_{1}\left(\varepsilon^{-1} \cdot\right) \ln \varepsilon+v_{2}\left(\varepsilon^{-1} \cdot\right)+\varepsilon z_{\varepsilon}\left(\varepsilon^{-1} \cdot\right)\right\|_{L^{2}(-\varepsilon, \varepsilon)}+|\zeta|\left\|u_{\varepsilon}\left(\varepsilon^{-1} \cdot\right)\right\|_{L^{2}(-\varepsilon, \varepsilon)} \\
\leq c_{1}\left(\varepsilon^{1 / 2}+\varepsilon^{1 / 4}\right)\|f\|+c_{2} \varepsilon^{1 / 2}|\ln \varepsilon|\left\|v_{1} \ln \varepsilon+v_{2}+\varepsilon z_{\varepsilon}\right\|_{L^{2}(\mathcal{I})} \\
+|\zeta| \varepsilon^{1 / 2}\left\|u_{\varepsilon}\right\|_{L^{2}(\mathcal{I})} \leq c \varepsilon^{1 / 4}\|f\|
\end{array}
$$


in view of Lemmas 3 and 4 . Here we also used inequality

$$
\int_{-\varepsilon}^{\varepsilon}\left|m\left(\frac{x}{\varepsilon}\right)\right|^{2} d x \leq \varepsilon \int_{-1}^{1}|m(t)|^{2} d t=\varepsilon\|m\|_{L^{2}(\mathcal{I})}^{2}
$$

for any $m \in L^{2}(\mathcal{I})$. Therefore (3.41) implies

$$
\left\|u_{\varepsilon}-y_{\varepsilon}\right\|=\left\|\left(H_{\varepsilon}-\zeta\right)^{-1} g_{\varepsilon}\right\| \leq|\zeta|^{-1}\left\|g_{\varepsilon}\right\| \leq c \varepsilon^{1 / 4}\|f\| .
$$

Now let us consider the difference

$$
u_{\varepsilon}(x)-u(x)= \begin{cases}\rho_{\varepsilon}(x) & \text { if }|x|>\varepsilon, \\ v_{0}\left(\frac{x}{\varepsilon}\right)+v_{1}\left(\frac{x}{\varepsilon}\right) \varepsilon \ln \varepsilon+v_{2}\left(\frac{x}{\varepsilon}\right) \varepsilon+z_{\varepsilon}\left(\frac{x}{\varepsilon}\right) \varepsilon^{2}-u(x) & \text { if }|x|<\varepsilon .\end{cases}
$$

We can as before invoke bound (3.23), Lemmas 3 and 4 to derive

$$
\begin{aligned}
\left\|u_{\varepsilon}-u\right\| \leq\left\|\rho_{\varepsilon}\right\| & +\varepsilon^{1 / 2}\left\|v_{0}+v_{1} \varepsilon \ln \varepsilon+v_{2} \varepsilon+z_{\varepsilon} \varepsilon^{2}\right\|_{L^{2}(\mathcal{I})} \\
& +\|u\|_{L_{2}(-\varepsilon, \varepsilon)} \leq c_{1} \varepsilon^{1 / 2}\left(\|f\|+\max _{|x| \leq \varepsilon}|u(x)|\right) \leq c_{2} \varepsilon^{1 / 2}\|f\| .
\end{aligned}
$$

Recalling the definitions of $y_{\varepsilon}$ and $u$, we estimate

$$
\left\|\left(H_{\varepsilon}-\zeta\right)^{-1} f-(\mathcal{H}-\zeta)^{-1} f\right\|=\left\|y_{\varepsilon}-u\right\| \leq\left\|y_{\varepsilon}-u_{\varepsilon}\right\|+\left\|u_{\varepsilon}-u\right\| \leq C \varepsilon^{1 / 4}\|f\|,
$$

by (3.42) and (3.43). The last bound establishes the norm resolvent convergence of $H_{\varepsilon}$ to the operator $\mathcal{H}$ and estimate (1.10), which is the desired conclusion for the case when potential $V$ is resonant.

If $V$ is not resonant, function $u$ in asymptotics (3.2) solves problem (3.19). Since both the value $u(-0)$ and $u(+0)$ are equal zero, $u^{\prime}$ has no logarithmic singularity at the origin in view of Lemma 2. From this reason the uniform approximation to $y_{\varepsilon}$ has the form

$$
u_{\varepsilon}(x)= \begin{cases}u(x)+\rho_{\varepsilon}(x), & \text { if }|x|>\varepsilon, \\ \varepsilon v_{2}\left(\frac{x}{\varepsilon}\right)+\varepsilon^{2} z_{\varepsilon}\left(\frac{x}{\varepsilon}\right), & \text { if }|x|<\varepsilon,\end{cases}
$$

where $v_{2}$ solves the problem

$$
-v_{2}^{\prime \prime}+U v_{2}=0, \quad t \in \mathcal{I}, \quad v_{2}^{\prime}(-1)=-u^{\prime}(-0), \quad v_{2}^{\prime}(1)=u^{\prime}(+0) .
$$

The rest of the proof is similar to the proof for the previous case.

\section{REFERENCES}

[1] R. Loudon. One-dimensional hydrogen atom. American Journal of Physics 27(9) (1959), 649-655.

[2] L. K. Haines, D. H. Roberts. One-dimensional hydrogen atom. American Journal of Physics 37(11), (1969), 1145-1154.

[3] Andrews, M. Singular potentials in one dimension. American Journal of Physics 44(11), (1976), 1064-1066.

[4] C. H. Mehta, S. H. Patil. Bound states of the potential $V(r)=-\frac{Z}{(r+\beta)}$. Physical Review A 17(1), (1978), 43-46.

[5] F. Gesztesy. On the one-dimensional Coulomb Hamiltonian. Journal of Physics A: Mathematical and General 13(3), (1980), 867.

[6] M. Klaus. Removing cut-offs from one-dimensional Schrödinger operators. Journal of Physics A: Mathematical and General 13(9), (1980), L295. 
[7] U. Oseguera and M. de Llano, Two singular potentials: the space-splitting effect. Journal of Mathematical Physics 34, 4575 (1993).

[8] M. Moshinsky. Penetrability of a one-dimensional Coulomb potential. Journal of Physics A: Mathematical and General 26 (1993), 2445-2450.

[9] R. G. Newton. Comment on 'Penetrability of a one-dimensional Coulomb potential' by $M$ Moshinsky. Journal of Physics A: Mathematical and General 27 (1994), 4717-4718.

[10] M. Moshinsky. Response to "Comment on 'Penetrability of a one-dimensional Coulomb potential" " by Roger G Newton. Journal of Physics A: Mathematical and General 27 (1994), 4719-4721.

[11] W. Fischer, H. Leschke and P. Müller. The functional-analytic versus the functionalintegral approach to quantum Hamiltonians. The one-dimensional hydrogen atom. Journal of Physics A: Mathematical and General 36 (1995), 2313-2323.

[12] P. Kurasov. On the Coulomb potential in one dimension. Journal of Physics A: Mathematical and General 29 (1996) 1767-1771.

[13] W. Fischer, H. Leschke and P. Muller. Comment on 'On the Coulomb potential in one dimension' by P Kurasov. Journal of Physics A: Mathematical and General 30 (1997) 55795581.

[14] P. Kurasov. Response to "Comment on 'On the Coulomb potential in one dimension" " by Fischer, Leschke and Muller. Journal of Physics A: Mathematical and General 30 (1997) 5583-5589.

[15] C. R. de Oliveira and A. A. Verri, Self-adjoint extensions of Coulomb systems in 1, 2 and 3 dimensions. Annals of Physics 324 (2009) 251-266.

[16] B. Bodenstorfer, A. Dijksma and H. Langer. Dissipative eigenvalue problems for a SturmLiouville operator with a singular potential. Proceedings of the Royal Society of Edinburgh Section A: Mathematics 130(6) (2000), 1237-1257.

[17] Yu. D. Golovaty, R. O. Hryniv. On norm resolvent convergence of Schrödinger operators with $\delta^{\prime}$-like potentials. Journal of Physics A: Mathematical and Theoretical 43 (2010) 155204 (14pp) (A Corrigendum: 2011 J. Phys. A: Math. Theor. 44 049802)

[18] Yu. Golovaty. Schrödinger operators with $\left(\alpha \delta^{\prime}+\beta \delta\right)$-like potentials: norm resolvent convergence and solvable models, Methods of Funct. Anal. Topology (3) 18 (2012), 243-255.

[19] Yu. D. Golovaty and R. O. Hryniv. Norm resolvent convergence of singularly scaled Schrödinger operators and $\delta^{\prime}$-potentials. Proceedings of the Royal Society of Edinburgh: Section A Mathematics 143 (2013), 791-816.

[20] Yu. Golovaty, 1D Schrödinger Operators with Short Range Interactions: Two-Scale Regularization of Distributional Potentials. Integral Equations and Operator Theory 75(3) (2013), 341-362.

[21] A. V. Zolotaryuk. Two-parametric resonant tunneling across the $\delta^{\prime}(x)$ potential. Adv. Sci. Lett. 1 (2008), 187-191.

[22] A. V. Zolotaryuk. Point interactions of the dipole type defined through a three-parametric power regularization. Journal of Physics A: Mathematical and Theoretical 43 (2010), 105302.

[23] Yu. Golovaty. Two-parametric $\delta^{\prime}$-interactions: approximation by Schrödinger operators with localized rank-two perturbations. Journal of Physics A: Mathematical and Theoretical 51(25) (2018), 255202.

[24] Yu. Golovaty. Schrödinger operators with singular rank-two perturbations and point interactions. Integr. Equ. Oper. Theory 90:57 (2018).

Department of Mechanics and Mathematics, Ivan Franko National University of LVIV, 1 Universytetska STR., 79000 LVIV, UkRAine

E-mail address: yuriy.golovaty@lnu.edu.ua 